\title{
Seminare 2009
}

\section{Praxiscomputerworkshop}

\section{Inhalt}

Die Workshopteilnehmer/innen erhalten im 1. Teil eine Einführung in die Anforderungen an ein Praxisinformationssystem. Anhand einer modernen vernetzten Praxisinfrastruktur werden die Beurteilungskriterien für eine praxis- und zukunftstaugliche Softwarelösung dargestellt. Checklisten sollen die schnelle Orientierung unterstützen und bei der Beurteilung und Wahl des Produkts konkrete Hilfe bieten. In Zusammenarbeit mit SGAM.Informatics werden die zentralen Elemente der elektronischen Krankengeschichte aufgezeigt. Ein Erfahrungsbericht eines EDV-Anwenders (Arzt) rundet den 1. Teil ab. Der 2. Teil umfasst die Präsentation von sechs Praxisadministrationssoftwarelösungen (Leistungserfassung, elektronisches Abrechnen unter Einbezug der TrustCenter, Agendaführung, Statistiken, Laborgeräteeinbindung, elektronische Krankengeschichte, Finanzbuchhaltungslösungen usw.).

\section{Ziel}

Die Teilnehmer/innen erhalten einen Anforderungskatalog, welcher ihnen erlaubt, ihre Vorstellungen für ein modernes Praxisinformationssystem besser $\mathrm{zu}$ formulieren und diese dem Softwarehersteller zu dessen Vorbereitung zu kommunizieren. Zudem erhalten sie einen ersten Überblick über führende Softwarelösungen.

\section{Daten}

$\begin{array}{lll}\text { K13 Donnerstag, 26. März 2009 } & \text { Techno- } \\ \text { Zürich } & 9.30-16.30 \mathrm{Uhr} & \text { park }\end{array}$

Folgende Softwareanbieter können Sie im 2. Teil des Workshops kennenlernen:

\section{Ärztekasse, Urdorf (CB 6)}

Als Marktleader seit 1964 im Bereich Praxisadministration können Sie bei der Ärztekasse auf eine ganzheitliche Lösung für Ihre Fragen betreffend Abrechnungsvarianten, Computerwahl, Netzwerke, Formular- und Briefbearbeitung usw. zählen. Die statistischen Erhebungen (im Auftrag der FMH Roko) sind ein anerkanntes betriebswirtschaftliches und standesorganisatorisches Hilfsmittel für die moderne Praxisführung. Nebst innovativen Ideen und Lösungen sowie Kooperationspartnerschaft mit 10 TrustCenter ist uns eine umfassende Kundenbetreuung ein Anliegen. Unsere Standorte befinden sich in Basel, Bern, Chur, Crissier, Genf, Le Landeron, Lugano, Luzern, St. Gallen, Thônex und Zürich. Sie definieren Ihre Wünsche - wir bieten Ihnen die Lösung!

\section{InfoCall Produkte AG, Zug (MEDICOwin ${ }^{\circledR}$ )}

Kompetenz, Qualität und Kontinuität sind seit mehr als 25 Jahren Leitspruch unserer Firma. Wir beraten anspruchsvolle Kunden in allen Belangen der Informationstechnologie. Unsere modular einsetzbare Software wird mit modernsten Werkzeugen nach neusten Erkenntnissen und Erfahrungen unserer Anwender entwickelt. Betriebssichere und bedienerfreundliche Gesamtlösungen garantieren so für optimale Wertschöpfung und lange Lebensdauer. Mit über 3000 täglich im Einsatz stehenden Programmmodulen zählen wir zu den erfolgreichen Softwarehäusern in der Schweiz. Mit MEDICOwin ${ }^{\circledR}$ offerieren wir als erste Firma in der Schweiz die elektronische KG mit integrierter Spracherkennung und den Export der gesamten Krankengeschichte auf einen biometrisch geschützten Stick. Falls der Patient dem Arzt die Erlaubnis gibt, kann dieser die KG lesen und mit MEDICOwin ${ }^{\circledR}$ importieren. Ebenfalls als eine der Ersten wurden wir für die volle Integration der Covercard zertifiziert. Unser Migrationsprogramm MEDICO ${ }^{\mathrm{cv}}$ erlaubt Ärztinnen und Ärzten, die heute andere Softwareprodukte einsetzen, einen problemlosen Umstieg auf MEDICOwin ${ }^{\circledR}$.

\section{Kern Concept AG, Gossau SG (AESKULAP)} AESKULAP ist ein extrem effizientes Administrationsprogramm, das zusammen mit einer SQLDatenbank höchste Verarbeitungsgeschwindigkeit, Kompaktheit und schnelle Zugriffszeiten gewährleistet. AESKULAP bietet für jedes Budget und für alle Bedürfnisse eine optimale und intelligente Lösung: vom einfachen Abrechnungssystem mit sämtlichen Leistungserfassungsmöglichkeiten bis hin zur führenden, vollstrukturierten elektronischen KG-Führung, die in der Praxis keine Wünsche offenlässt. AESKULAP ist ein modular aufgebautes und anpassbares System mit einer Vielzahl von Modulen. Unsere extrem preisgünstige Basisversion kann somit bei Bedarf jederzeit erweitert werden. AESKULAP - die intelligente Ärztesoftware für jedes Budget! 


\section{TMR - Triangle Micro Research AG, Hölstein (WinMed ${ }^{\circledR}$ )}

Die TMR AG wurde von Ärzten, Wissenschaftlern, Unternehmern und Dozenten der Uni Basel 1993 als «Spin-Off»-Firma gegründet. Der Schwerpunkt in der Entwicklung der TMR AG bilden Anwendungen im Bereich der med. Telekommunikation, Internet sowie der mehrmandanten- und mehrplatzfähigen Ärztesoftware WinMed ${ }^{\circledR}$. Win$\mathrm{Med}^{\circledR}$ ist ein vollständiges, äusserst einfach zu bedienendes Arztpraxisadministrationssystem mit integrierter Kommunikationsplattform, Bild- und Dokumentenverwaltung sowie modernster elektronischer Krankengeschichte. WinMed ${ }^{\circledR}$ wird nach ausgereifter mehrjähriger Pilotphase erfolgreich seit 1998 verkauft und zählt heute zu den meistgekauften Ärztesoftwarepaketen. Vertrieben wird WinMed ${ }^{\circledR}$ in der Deutschschweiz von TMR AG selbst und im Tessin von GFP Mediconsul in Massagno.

\section{Triamun AG, Gümligen (TriaMed ${ }^{\circledR}$ )}

Triamun entwickelt und vermarktet innovative Softwarelösungen für Praxis- und Apothekenmanagement. Individuelle Beratung, die gesamte Soft- und Hardware, einen umfassenden Support und Schulung aus einer Hand. Die Produkte und Dienstleistungen auf der Plattform .NET bieten für jedes Bedürfnis die geeignete Lösung. Die Softwarelösungen für Arztpraxen basieren auf modernster Technologie und wurden zusammen mit Ärzten entwickelt. So sind intuitiv bedienbare Managementlösungen für Einzel- und Gruppen- praxen sowie Netzwerke entstanden, die sämtliche Bedürfnisse nach integriertem und vernetztem Arbeiten befriedigen. Die Softwarelösungen auf .NET-Plattform, mit ihren Zusatzmodulen für die Arztpraxis, wurden während 20 Jahren den Kundenbedürfnissen ständig angepasst, so dass sie heute als modernste lokale Client-ServerLösung oder zentrale Terminal Server-Lösung optimal auf die Abläufe in der Arztpraxis zugeschnitten ist.

\section{vitodata AG, Ohringen b. Winterthur} (vitoMed Administrationssystem)

Die vitodata AG besteht seit mehr als 26 Jahren. Die Unternehmung konzentriert sich auf Praxisund Kliniklösungen. Die innovative Haltung eröffnet laufend neue Einsatzgebiete - immer mit dem entsprechenden Nutzen für die Anwender. In den ersten 25 Jahren stand die Abrechnung mit der ICT im Vordergrund. Heute ist die vitodata AG auch bei der elektronischen Krankengeschichtenführung an der Spitze im Schweizer Markt. Die Marktführerschaft ist für das ganze Team der vitodata AG eine Verpflichtung, im Sinne des Investitionsschutzes für die Kunden den Fortbestand zu sichern und unternehmerisch und ethisch korrekt zu handeln.

\section{Anmeldung und Auskunft}

Internet: www.fmhservices.ch oder FMH Consulting Services, Sandra Stadelmann, Burghöhe 1, 6208 Oberkirch, Tel. 04192500 77, Fax 041 9210586.

\section{G'FMH INKASSO}

\section{Säumige Patienten?}

Kommt Ihnen folgender Ablauf bekannt vor?

1. Versand Honorarrechnung - Zahlungsziel 30 Tage

2. Versand 1. Mahnung - Zahlungsziel 7 Tage

3. Versand 2. Mahnung - Zahlungsziel 7 Tage

4. Versand 3. Mahnung - Zahlungsziel 7 Tage

5. Betreibungsandrohung

Seien Sie sich bewusst, dass, wenn Ihre Honorarforderung nach Rechnungsstellung und 1. Mahnung noch nicht beglichen ist, Sie auf eine 2. und 3. Mahnung verzichten können. Ab 2. Mahnung sollten Sie ein Zeichen setzen und die Angelegenheit einem Inkassospezialisten übergeben. Nun weiss der säumige
Schuldner, dass ein Profi am Werk ist! Als marktführendes Inkassounternehmen für Ärzte und Zahnärzte stehen wir Ihnen gerne zu Diensten.

\author{
Unser Angebot umfasst: \\ - Bonitätsprüfung (online via my.inkas) \\ - Vorrechtliches Inkasso \\ - Rechtliches Inkasso \\ - Verlustscheininkasso
}

Senden Sie uns Ihre Rechnungskopie mit Einzahlungsschein oder die dritte Mahnung! Den Rest übernehmen wir!

\section{FMH Inkasso Services}

Inkassostelle ENCATH AG $\bullet$ Koordinationsstelle

Postfach $800 \bullet 2501$ Biel

Telefon $0323443969 \bullet$ Fax 0323443966

mail@fmhinkasso.ch • www.fmhinkasso.ch 